\title{
Identification of Barriers in Onco-Physiotherapy: Patient's Perspective; A Cross-Sectional Observational Study
}

\author{
Hetanshi Raikundlia ${ }^{1}$, Siddhi Ghodge ${ }^{2}$ \\ ${ }^{1}$ Intern, K. J Somaiya College of Physiotherapy, Mumbai. \\ ${ }^{2}$ Assistant Professor, Department of Cardiorespiratory Sciences, K. J. Somaiya College of Physiotherapy, \\ Mumbai. \\ Corresponding Author: Hetanshi Raikundlia
}

\begin{abstract}
Cancer is a debilitating and complex disease that affects millions of people around the world, it has been identified that cancer rehabilitation including physiotherapy can be important factor in management of cancer patients and survivors.

There are disquisitions that showcase and prove the presence of certain problems with respect to referral and delivery of the physiotherapy treatment given to oncology patients. It is noticed that in spite of available facilities a large number of our population including the cancer patients are unaware of the presence of onco-physiotherapy and its potential benefits. This cross sectional study conducted among cancer patients aims to identify these barriers to help gain a complete understanding of them and the benefits of rehabilitation will help us gain valuable insight to optimise the beneficial services and its deliverance at these centres.

It showed that only half of the samples were aware of onco-physiotherapy. There are multiple barriers faced especially with respect to the referral system present in our country. Though they do not lack motivation, family and community support, ability to follow exercise regime and home programme, this study showed that in spite of this being conducted in an urban setting most were unaware of such services. This tells us that there is a dire need of awareness among patients, doctors and general population. There is also a need for more awareness about NGOs, government schemes and specialised branch of onco-physiotherapy to aid the rehabilitation process.
\end{abstract}

Keywords: Oncology, cancer rehabilitation, onco-physiotherapy, barriers, awareness.

\section{INTRODUCTION}

Cancer is one of the leading health problems faced in India. $70 \%$ of the cancers occur between the ages of 30 to $69^{1}$, despite vast improvements in developing potential treatments and abundant funding into research for devising potential cures.

However, it is important to observe that the survival rate for cancer patients has been bolstered by early diagnosis along with advanced treatments. Moreover, Cancer rehabilitation including physiotherapy has been identified as an important factor in management of cancer survivors.
It not only enhances the physical \& psychological condition of the survivor but also improves their engagement with the population. $^{2}$

Cancer rehabilitation can be preventive, restorative, supportive and palliative. ${ }^{3}$ There are studies that have been conducted which prove that rehabilitation services including physical therapy, followed by physical training, psychological counselling, supportive group sessions, admittance to a convalescent home, consultation with social worker and 
Hetanshi Raikundlia et.al. Identification of barriers in onco-physiotherapy: patient's perspective; a crosssectional observational study.

occupational therapy are needed by cancer patients. ${ }^{4}$

Studies also elucidate that there is a definite improvement not only in the quality of life and functional levels but also helps develop a positive effect on the length of hospital stay, fatigue levels, pain and appetite associated with cancer treatments as a result of receiving appropriate physiotherapy at the correct time ${ }^{5}$

Patients that undergo different forms of cancer treatment such as chemotherapy or radiation have a high chance of developing cardiovascular and pulmonary problems which not only require physiotherapy interventions but also consistent monitoring throughout treatment. ${ }^{6}$

There are disquisitions that showcase and prove the presence of certain problems with respect to referral and delivery of the physiotherapy treatment given to oncology patients. Along with this, it was noted that the number of available physiotherapists is not commensurable with the increasing needs for services by cancer patients. Thereby placing an increased demand for qualified physiotherapy professionals and adequately equipped departments to render these services. ${ }^{7}$

It is noticed that in spite of available facilities a large number of our population including the cancer patients are unaware of the presence of onco-physiotherapy and its potential benefits, hence this study aims to identify the various barriers in oncophysiotherapy faced by patients. Along with this the study compares awareness of oncophysiotherapy with gender and types of various to get an even more better understanding of this significant barrier.

In spite of existing facilities for onco-physiotherapy there are quite a few barriers which need to be identified and overcome in order to provide optimum services towards recovery.

A complete understanding of the barriers and benefits of rehabilitation will help us gain valuable insight to optimise the beneficial services and its deliverance at these centres. This is why it is imperative for us to investigate all the challenges faced by the cancer patients with respect to the existing rehabilitation facilities and to devise solutions in order to overcome them, especially in a country like India where awareness of the existence of these rehabilitation facilities is limited.

\section{METHODOLOGY}

It was a cross-sectional observational study conducted among cancer patients (January-June 2020). Ethical approval was obtained from the institutional review board of K.J. Somaiya college of Physiotherapy.

A questionnaire was developed denovo as a part of this study. It was designed to identify barriers faced in oncophysiotherapy from the patients' perspective. It was validated from oncophysiotherapy practioners and representative population. Medical terminologies were avoided as much as possible.

Patients with any type of cancer who had been diagnosed for more than 6 months were invited to participate in this study. Participation was voluntary and e-consent was obtained from each of them. Patients with a mini mental state examination score of less than 24 were not included. The questionnaire aimed to check respondents awareness about onco-physiotherapy, what barriers do they face while accessing it and to help understand where the issue lies in order to rectify them and optimise these services. Comparison of awareness was done with gender and the type of cancer to get a deeper understanding. The questionnaire was distributed to the participants as Google forms via social media on WhatsApp; and was emailed, if requested by them.

All the responses obtained via Google-forms were screened. Inaccurate or incomplete responses were discarded. The close-ended data was analysed automatically using Google spread- sheet and descriptive statistics using percentage and frequency distribution was performed. 
Hetanshi Raikundlia et.al. Identification of barriers in onco-physiotherapy: patient's perspective; a crosssectional observational study.

The open-ended questions were analysed using categorization and thematic analysis.

\section{RESULTS}

It was seen that the majority of the respondents were females, $62.7 \%$.

Most reported type of cancer was CA breast (37.3\%) while the least reported ones were CA oral and CA stomach (2.0\%).

Table no. 1 and pie chart no. 1 show that only $57.8 \%$ of the respondents were aware of onco-physiotherapy.

Table no. 1. Awareness of onco-physiotherapy.

\begin{tabular}{|l|l|}
\hline RESPONSE & PERCENTAGE (\%) \\
\hline Yes & 57.8 \\
\hline Not sure & 9.8 \\
\hline No & 32.4 \\
\hline
\end{tabular}

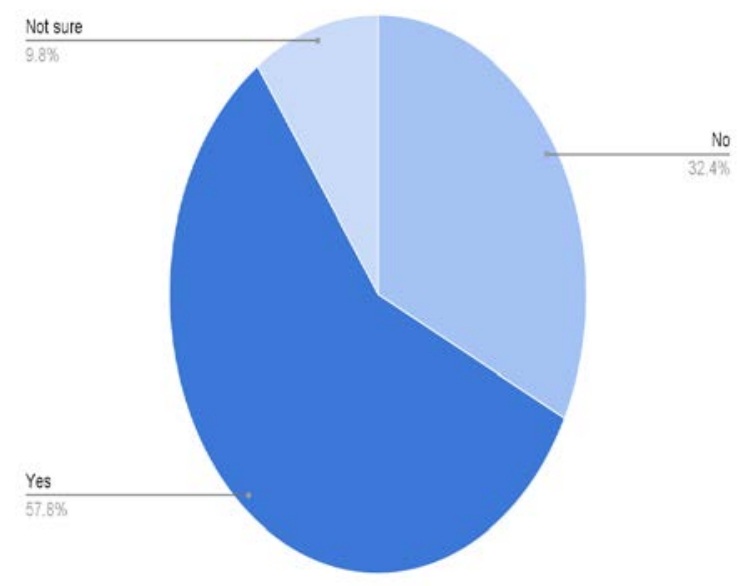

Pie chart no. 1. Awareness of onco-physiotherapy.

Table no. 2. Doctors referral.

\begin{tabular}{|l|l|}
\hline \multicolumn{2}{|c|}{ Table no. 2. Doctors referral. } \\
\hline After diagnosis & PERCENTAGE (\%) \\
\hline During chemotherapy/radiotherapy. & 14.7 \\
\hline After surgery & 16.7 \\
\hline
\end{tabular}

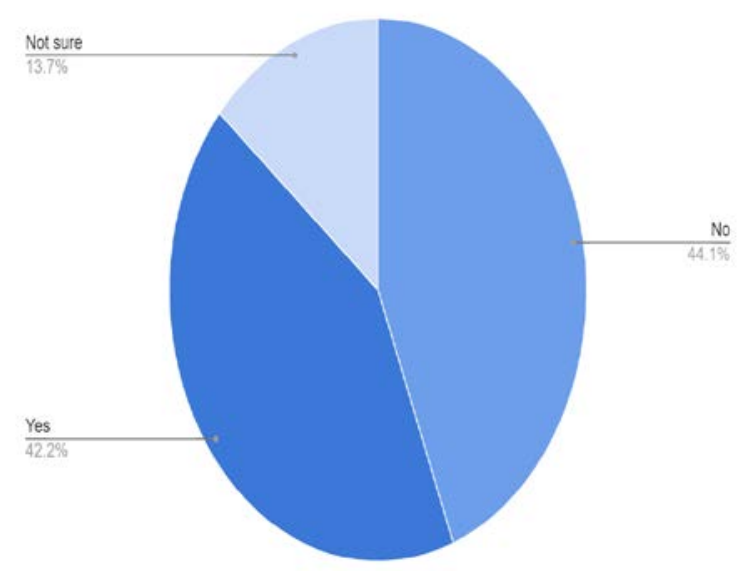

Pie chart no. 2. Awareness of onco-physiotherapy set up near them.
Table no. 3. Awareness of onco-physiotherapy set up near them.

\begin{tabular}{|l|l|}
\hline RESPONSE & PERCENTAGE (\%) \\
\hline Yes & 42.2 \\
\hline Not sure & 13.7 \\
\hline No & 44.1 \\
\hline
\end{tabular}

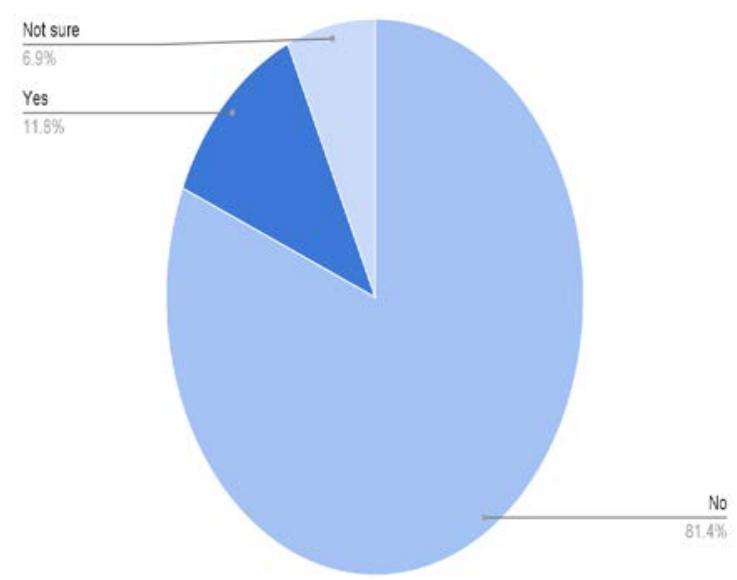

Pie chart no. 3. Awareness of NGOs/government schemes.

Tabe no. 4. Awareness of NGOs/government schemes.

\begin{tabular}{|l|l|}
\hline RESPONSE & PERCENTAGE (\%) \\
\hline Yes & 11.8 \\
\hline Not sure & 6.9 \\
\hline No & 81.4 \\
\hline
\end{tabular}

Table no. 5. Awareness of onco-physiotherapy among different types of cancer.

\begin{tabular}{|l|l|}
\hline $\begin{array}{l}\text { TYPE OF } \\
\text { CANCER }\end{array}$ & $\begin{array}{l}\text { PERCENTAGE } \\
\text { AWARENESS }\end{array}$ \\
\hline Luekemia & 100 \\
\hline CA pancreas & 100 \\
\hline Lymohoma & 100 \\
\hline CA thyroid & 100 \\
\hline CA lung & 85.7 \\
\hline Hodgkin lymphoma & 50 \\
\hline CA ovary & 50 \\
\hline CA tongue & 50 \\
\hline CA buccal mucosa & 50 \\
\hline CA oesophagus & 50 \\
\hline CA colon & 33.3 \\
\hline CA rectum & 33.3 \\
\hline
\end{tabular}

When asked at which stage do you think onco-physiotherapy should be start, $71.6 \%$ reported with the response of after surgery, $13.7 \%$ reported after diagnosis, 9.8\% reported after chemotherapy and $4.9 \%$ reported with after radiotherapy.

It was observed that only $14.7 \%$ of the respondents were given a reference by their doctors for onco-physiotherapy after diagnosis, $16.7 \%$ were given a reference during chemotherapy/radiotherapy. $77.5 \%$ were given a reference after surgery, as indicated in table no. 2.

$82.4 \%$ reported with a 'NO', $13.7 \%$ reported with 'NOT SURE' and 3.9\% 
Hetanshi Raikundlia et.al. Identification of barriers in onco-physiotherapy: patient's perspective; a crosssectional observational study.

reported with a 'YES' when asked if they were afraid that their disease might worsen after exercise.

$88.2 \%$ of them reported affirmatively when asked if they thought physiotherapy was important for their recovery.

$70.6 \%$ were given some verbal/printed protocol for the exercises to be done, out of which $93.1 \%$ of them were able to understand it.

Only $42.2 \%$ of them were aware of availability of any such services near them as shown in pie chart no. 2 and table no. 3 .

Out of these only $53.5 \%$ of them had visited the set up.

$60.8 \%$ of the respondents indicated towards having a convenient mode of transportation to avail physiotherapy services.

$26.5 \%$ of the respondents reported to have issues related to transportation cost. affordable.

Only $61.8 \%$ found cost of treatment

$52.9 \%$ reported that they required a family member to take them to a rehabilitation centre.

Majority of the respondents, $98 \%$ of them, reported affirmatively when asked about family support.

When asked about community support, $82.4 \%$ of them reported affirmatively.

$76.5 \%$ reported no when asked if they lack self-motivation when it comes to availing physiotherapy services.

$80.4 \%$ were able to cope with the exercises given.

$67.6 \%$ of them were able to follow through with the home exercise programme given to them.

$81.4 \%$ were unaware of any NGOs/Government schemes as indicated in table no. 4 and pie chart no. 3 .

When comparing awareness of oncophysiotherapy with gender, it was seen that $62.5 \%$ of the females and $50 \%$ of the males that responded were aware of oncophysiotherapy.
When comparing awareness of oncophysiotherapy with various types of cancer it was seen that among our population those having CA breast, $71.8 \%$ were aware of onco-physiotherapy.

Table no. 5 shows awareness of onco-physiotherapy among different types of cancer identified in this study.

Respondents with CA caecum, CA stomach, CA testicles, CA uterus, CA gall bladder, CA head and neck, CA ileum, CA lower lip and Ewing sarcoma had 0\% awareness about onco-physiotherapy.

\section{DISCUSSION}

This study was conducted among cancer patients to get their perspective and gain an understanding on what changes could be made for the betterment of the rehabilitation services provided to them.

We received 102 responses, the data collected was then screened and analysed. The population which participated in the study falls in the age group of 25-80. The population which was included in the study when analysed on type of cancer included $37.3 \%$ of breast cancer, $9.8 \%$ of ovary cancer, $8.8 \%$ of buccal mucosa cancer and $6.9 \%$ of lung cancer and others. This again attributes to the data of GLOBOCAN 2020 India statistics which show that India has $13.5 \%$ of breast cancer prevalence, $10.3 \%$ of lip and oral cavity cancer, 3.5\% of ovary cancer and $5.5 \%$ of lung cancer. Considering the growing incidence in India it is of utmost importance to assess and remove barriers in onco-physiotherapy.

The common barriers assessed in our study were awareness, referral, fear of exercises, self-motivation, financial barrier, transportation, family and community support and we found out that:

\section{- Lack of awareness among cancer patients}

It was seen that only $57.8 \%$ of the respondents were aware of oncophysiotherapy, showing that still there is a large scope of increasing awareness. 
Hetanshi Raikundlia et.al. Identification of barriers in onco-physiotherapy: patient's perspective; a crosssectional observational study.

When asked at which stage they think onco-physiotherapy should start, $71.6 \%$ reported the response of after surgery, showing the lack of awareness of the effect and significance of oncorehabilitation.

Literature reviews show the importance and significant role of the physiotherapist in palliative care of a cancer patient, starting from the very beginning of their treatment and then following through as an imminent member of the multidisciplinary team at the hospital level of care. ${ }^{15}$ Studies suggest how an effectual prerehabilitative exercise protocol can help in the reduction of duration of stay in the hospital and post-surgery impediments along with improvement in quality of life and speed of recovery. ${ }^{16}$ Research shows how it not only helps deal with physical aspects such as fatigue, strength, respiratory capacity but also emotional aspects keeping in mind their mental state which may be affected due to this debilitating disease. ${ }^{17}$ Based on a survey conducted among a large population of cancer survivors in a developed country it was seen that there were a significant number of health consequences which interfered with their quality of life. Cancer rehab for them could prove beneficial. ${ }^{18}$

\section{- Referral System in India}

It is always a team approach which needs to be achieved. However, our study showed that only $14.7 \%$ of the respondents were given a reference by their doctors for onco-physiotherapy after diagnosis, $78.7 \%$ were $16.7 \%$ given a reference during chemotherapy/radiotherapy.

$77.5 \%$ were given a reference after surgery, which indicates the variety of views of oncologists and onco-surgeons.

A number of researches suggest that an integrative approach would be beneficial to the patient in terms of providing them with a chance to keep up with the different aspects of their treatment. ${ }^{19}$ There are studies which show that although physicians are aware of onco-rehabilitation there is a low referral rate which is one of the major barriers faced by these patients. ${ }^{20}$

Literature search also shows a major difference in attitude between members of palliative care with respect to cancer rehabilitation ${ }^{21}$ and how physician centered barriers such as hesitancy to refer due to their view that rehabilitation can interfere with their treatment indicates how more awareness needs to be created about oncorehabilitation and its various benefits to the patient. $^{22}$

Thus, lack of awareness amongst doctors is an important barrier identified by this study.

This can be overcome by educating and creating awareness among practitioners and the general population about the specialised branch of onco-physiotherapy.

\section{- Communication barrier}

This was not found to be a barrier but there is definite scope for improvement.

\section{- Awareness and presence of a rehabilitation set up near by}

$42.2 \%$ of them were aware of availability of any such services near them, hence indicating the possibility of lack of awareness and/or presence of an oncophysiotherapy setup.

Out of these only $53.5 \%$ had visited one.

Thus, there is a need for more such setups and need to increase awareness about the same.

\section{- Monetary issues- including travel and service charges.}

$26.5 \%$ of the respondents reported to have issues related to transportation cost.

Only $61.8 \%$ found the cost of treatment affordable, indicating how money can be a barrier for a large number of cancer patients.

However, literature suggests that there are major economic problems faced by cancer patients in our country hence this requires more research on an in-depth level and larger scale to develop and improve 
Hetanshi Raikundlia et.al. Identification of barriers in onco-physiotherapy: patient's perspective; a crosssectional observational study.

government intervention in order to bear costs of the treatment by these patients. Rehabilitation services can be included under health insurance cover as these are lifelong treatment regimens required for certain long term patient impairments. ${ }^{23,} 24$, 25

\section{- Awareness of NGOs/government schemes}

81.4\% were unaware of any NGOs or schemes. NGOs and such schemes can play an important role for cancer patients by various ways such as setting up early detection camps, monetary support and emotional support groups for patients and their families. ${ }^{26,27}$

Large numbers of NGOs all over the world play a significant role in dealing with the cancer burden in their country such as cancer council Australia, American cancer society etc. These NGOs publish periodicals to aid not only the patients but also the public in early detection of cancer symptoms and how to go about getting the best treatment. 28,29

\section{CONCLUSION}

This study showed that only $57.8 \%$ of the samples were aware of oncophysiotherapy. There are multiple barriers faced especially with respect to the referral system present in our country.

Though they do not lack motivation, family and community support, ability to follow exercise regime and home programme, thus study showed that a large percentage of the others are not aware of such services in spite of this study being conducted in an urban setting. This tells us that there is a dire need of awareness among patients, doctors and general population.

We encountered some limitations in our project, analysis of different types of cancer and the stages of cancer could not be done in this study. Sample size was restricted to an urban population.

It throws light on how there is a need to further increase awareness about oncophysiotherapy among not only cancer patients but also members of the palliative care as well as onco-practitioners.

This study shows clear indication towards the need for more inclusion of physiotherapy in the multidisciplinary approach of treatment for a cancer patient. The study highlights a lack in the referral system. Having a more collaborative effort and improved teamwork between palliative care and onco-rehabilitation can help treat them in a simultaneous and effective manner.

More awareness can be brought about in all types' cancer and not restricted to the ones commonly occurring. Research done from different perspectives such as that from the family or the healthcare members involved at different levels can help gain a deeper understanding of the barriers.

An individual viewpoint of the patient and caregiver varies in India depending on their ethnicity and cultural diversity. Each individual perceives their impairment differently and thus it is the responsibility of the health care professionals to bring about an integrated approach for cancer rehabilitation.

This being a disease with high mortality and morbidity as compared to other diseases it is necessary to provide all the possible supportive care to these individuals. Hence there is need for more awareness about NGOs, government schemes and specialised branch of oncophysiotherapy to aid the rehabilitation process.

\section{ACKNOWLEDGEMENT}

The authors would like to acknowledge the valuable contribution of the cancer patients that participated and support of the principal and faculty of the institute in the conduction of this study.

Conflict of Interest: The authors declare that they have no conflict of interests. 
Hetanshi Raikundlia et.al. Identification of barriers in onco-physiotherapy: patient's perspective; a crosssectional observational study.

Funding: The authors have not received any kind of financial support in the conduct or publication of this research.

\section{Ethical Approval: Approved}

\section{REFERENCES}

1. Dikshit R, Gupta PC, Hettige CR, Gajalakshmi V, Aleksandrowicz L, Rajendra Badwe, et al. Cancer mortality in India: a nationally representative survey. The Lancet. 2012; 379(9828): 1807-16.

2. Eun Joo Yang, Seung Hyun Chung, JaeYong Jeon, Kwan Sik Seo, Hyung-Ik Shin, Ji Hye Hwang, and Jae-Young Lim. Current Practice and Barriers in Cancer Rehabilitation: Perspectives of Korean Physiatrists. Cancer Res Treat. 2015; 47(3): 370-378.

3. Karthikeyan G, Udaya Kumar Manoor, Sanjay Sudakar Supe. Development and Content validation of Questionnaires to Examine Current Status of Physical Therapy Practice in Cancer Rehabilitation in South India. J Phys Ther. 2012; 6(4): 50-60.

4. Thorsen L, Gjerset GM, Loge JH, Kiserud CE, Skovlund E, Fløtten T, Fosså SD. Cancer patients' needs for rehabilitation services. Acta Oncol 2011; 50(2):212-22.

5. Laakso EL, McAuliffe AJ, Cantlay A. The impact of physiotherapy intervention on functional independence and quality of life in palliative patients. Cancer Forum 2003; 27(1): 15-20.

6. Packel L. Cardiopulmonary Implications of Cancer Treatments. Cardiopulmonary Physical Therapy Journal. 2003; 14(2): 103.

7. Karthikeyan Guru, Udayakumar Manoor, Sanjay S Supe. Physiotherapy services for cancer patients in South India: A survey, Physiotherapy and Occupational Therapy Indian Journal of Physiotherapy \& Occupational Therapy. 2018; 12(1): 22-29.

8. Samantha Hendren, MD, MPH, Nancy Chin, PhD, MPH, Susan Fisher, PhD, Paul Winters, MS, Jennifer Griggs, MD, MPH, Supriya Mohile, MD, and Kevin Fiscella, MD, MPH. Patients' Barriers to Receipt of Cancer Care, and Factors Associated with Needing More Assistance from a Patient Navigator. J Natl Med Assoc. 2011; 103(8): 701-710.
9. Lehmann JF, DeLisa JA, Warren CG, deLateur BJ, Bryant PL, Nicholson CG. Cancer rehabilitation: assessment of need, development, and evaluation of a model of care. Arch Phys Med Rehabil. 1978; 59(9) :410-9.

10. Riccardo E Marioni, Mark Chatfield, Carol Brayne,Fiona E Matthews \& Medical Research Council Cognitive Function and Ageing Study, The reliability of assigning individuals to cognitive states using the Mini Mental-State Examination: a population-based prospective cohort study. BMC Medical Research Methodology. 2011; 11(1): 127.

11. Raja Paramjeet Singh Banipal, Harminder Singh, and Baltej Singh, Assessment of Cancer-related Fatigue among Cancer Patients Receiving Various Therapies: A Cross-sectional Observational Study, Indian J Palliat Care. 2017; 23(2): 207-211.

12. Santa Mina D, Clarke H, Ritvo P, Leung YW, Matthew AG, Katz J, Trachtenberg J, Alibhai SM. Effect of total-body prehabilitation on postoperative outcomes: a systematic review and meta-analysis. Physiotherapy. 2014; 100(3): 196-207.

13. Andrea Cheville, Rehabilitation of Patients with Advanced Cancer. Cancer. 2001; 92(4 Suppl): 1039-48.

14. Veronika Fialka-Moser, Richard Crevenna, Marta Korpan and Michael Quittan, Cancer rehabilitation. Particuarly with aspects on physical impairments. J Rehabil Med. 2003; 35(4): 153-162.

15. Colette Fulton. Physiotherapy In Cancer Care A Framework Of Rehabilitation Of Patients. Physiotherapy Journal. 1994; 8(12): 830-834.

16. Adamina M, Gié O, Demartines N, Ris F. Contemporary perioperative care strategies. Br J Surg. 2013 ;100(1): 38-54.

17. Lis Adamsen, Morten Quist, Christina Andersen, Tom Møller, Jørn Herrstedt, Dorte Kronborg, et al. Effect of a multimodal high intensity exercise intervention in cancer patients undergoing chemotherapy: randomised controlled trial. BMJ. 2009; 339: 3410.

18. Hewitt M, Rowland JH, Yancik R. Cancer survivors in the United States: age, health, and disability. J Gerontol A Biol Sci Med Sci. 2003; 58(1): 82-91.

19. Silver JK, Baima J, Mayer RS. Impairmentdriven cancer rehabilitation: an essential 
Hetanshi Raikundlia et.al. Identification of barriers in onco-physiotherapy: patient's perspective; a crosssectional observational study.

component of quality care and survivorship. CA Cancer J Clin. 2013; 63(5): 295-317.

20. Raúl A. Rosario-Concepción, Yailiz B. Calderín, Christian López Aponte, Carmen E. López-Acevedo, Fernando L. SepúlvedaIrrizarry. Oncologists' Attitude and Knowledge about Cancer Rehabilitation. PM R. 2021; 8(9): 192-193.

21. Spill GR, Hlubocky FJ, Daugherty CK. Oncologists' and physiatrists' attitudes regarding rehabilitation for patients with advanced cancer. PM R. 2012; 4(2): 96-108.

22. Kruser TJ, Kruser JM, Gross JP, Moran M, Kaiser K, Szmuilowicz E, Kircher SM. Medical oncologist perspectives on palliative care reveal physician-centered barriers to early integration. Ann Palliat Med. 2020; 9(5): 2800-2808.

23. Guruchanna Basavaiah, Priyanka D. Rent, Eugene G. Rent, Richard Sullivan, Margaret Towne, Marieke Bak, et al. Financial Impact of Complex Cancer Surgery in India: A Study of Pancreatic Cancer. J Glob Oncol. 2018; 4: 1-9.

24. Pandey AK, Vias P, Mangwana SK, Kaur S, Dimri K, Walia DK. The hospital-based socioeconomic study to assess the financial toxicity among cancer survivors. Clin Cancer Investig J. 2020; 9(4): 121-5.

25. Dinesh TA, Nair P, Abhijath V, Jha V, Aarthy K. Economics of cancer care: A community-based cross-sectional study in Kerala, India. South Asian J Cancer. 2020; 9(1): 7-12.

26. Gustavo Azenha, Loyce Pace Bass, Maira Caleffi, Robert Smith, Lauren Pretorius, Alessandra Durstine, et al. The role of breast cancer civil society in different resource settings. The Breast. 2011; 20(2 Suppl): 81-87.

27. Cecilia Vindrola Padros. The Participation Of NGOs In Healthcare: The Case Of Pediatric Cancer Treatment In Argentina. University of South Florida Scholar Commons Graduate Theses and Dissertations. 2009.

28. Xu T. Chinese Anti-Cancer Association as a non-governmental organization undertakes systematic cancer prevention work in China. Chin J Cancer Res. 2015; 27(4): 423-427.

29. Durstine A, Leitman E. Building a Latin American cancer patient advocacy movement: Latin American cancer NGO regional overview. Salud Publica Mex. 2009; 51(2 Suppl): 316-22.

How to cite this article: Raikundlia H, Ghodge $\mathrm{S}$. Identification of barriers in oncophysiotherapy: patient's perspective; a crosssectional observational study. Int J Health Sci Res. 2021; 11(11): 15-22. DOI: https://doi.org /10.52403/ijhsr.20211103 